\title{
Airborne field strength monitoring
}

\author{
J. Bredemeyer ${ }^{1}$, T. Kleine-Ostmann ${ }^{2}$, T. Schrader ${ }^{2}$, K. Münter ${ }^{2}$, and J. Ritter ${ }^{3}$ \\ ${ }^{1}$ FCS Flight Calibration Services GmbH, Braunschweig, Germany \\ ${ }^{2}$ Physikalisch-Technische Bundesanstalt, Braunschweig, Germany \\ ${ }^{3}$ EADS Deutschland GmbH, Military Aircraft, Bremen, Germany
}

\begin{abstract}
In civil and military aviation, ground based navigation aids (NAVAIDS) are still crucial for flight guidance even though the acceptance of satellite based systems (GNSS) increases. Part of the calibration process for NAVAIDS (ILS, DME, VOR) is to perform a flight inspection according to specified methods as stated in a document (DOC8071, 2000) by the International Civil Aviation Organization (ICAO). One major task is to determine the coverage, or, in other words, the true signal-in-space field strength of a ground transmitter. This has always been a challenge to flight inspection up to now, since, especially in the L-band (DME, 1GHz), the antenna installed performance was known with an uncertainty of $10 \mathrm{~dB}$ or even more. In order to meet ICAO's required accuracy of $\pm 3 \mathrm{~dB}$ it is necessary to have a precise 3-D antenna factor of the receiving antenna operating on the airborne platform including all losses and impedance mismatching. Introducing precise, effective antenna factors to flight inspection to achieve the required accuracy is new and not published in relevant papers yet. The authors try to establish a new balanced procedure between simulation and validation by airborne and ground measurements. This involves the interpretation of measured scattering parameters gained both on the ground and airborne in comparison with numerical results obtained by the multilevel fast multipole algorithm (MLFMA) accelerated method of moments (MoM) using a complex geometric model of the aircraft. First results will be presented in this paper.
\end{abstract}

\section{Introduction}

Preceding work was done describing the antenna installed performance of a flight inspection aircraft (Bredemeyer et al., 2004). Those investigations mainly focussed on the modelling of the platform and the antennas to be installed as a prerequisite to apply the method of moments (Harrington,

Correspondence to: J. Bredemeyer

(brd@ flightcalibration.de)
1968). Additionally, measurements of selected aircraft antennas were done in a GTEM cell to obtain the non-installed antenna factors and to form a model which was then used to improve the on-platform far field simulation. The final validation of the model should include measurements in a well-defined environment to derive the effective performance since one cannot implicitly rely on simulations only. The results and critical aspects are discussed below.

First, we need to describe the electric far field antenna factor (AF) which is given by (Kraus, 1988; Balanis, 1997):

$A F_{\text {electric }}=\frac{E_{\text {incident }}}{V_{\text {received }}}$

In case of a loss-less antenna with a gain $G$ and an impedance of $50 \Omega$ the ideal AF then is given for the wavelength $\lambda$ by

$A F_{\text {ideal }}=\frac{9.73}{\lambda \sqrt{G}}$.

The radiation pattern of an antenna is mainly determined by the shape and material of the radiating element, while impedance matching networks in the input section determine the input reflection coefficient $r$ of the antenna. DIN (DIN EN 45003, 1995) proposes the so called practical gain $G_{\text {pract }}$ to be used in the definition of the antenna factor of antennas with a specific gain $G_{0}$ in case of non-ideally matched antennas:

$G_{\text {pract }}=\left(1-|r|^{2}\right) G_{0}$.

In practice however, there are additional losses due to cable and connectors. An overall gain from the receiver's point of view must contain those values so that the effective gain can be written as

$G_{\text {eff }}=\left(1-|r|^{2}\right) \mathrm{e}^{-2 \alpha 1} \cdot \kappa \cdot G_{0}$

where $l$ denotes the cable length, $\alpha$ is the attenuation constant according transmission line theory (Unger, 1996), and $\kappa$ denotes the connector losses. Inserting $G_{\text {eff }}$ as $G$ in Eq. (2) then leads to the effective antenna factor:

$A F_{\text {eff }}=\frac{9.73}{\lambda \sqrt{\left(1-|r|^{2}\right) \cdot \kappa \cdot G_{0}}} \cdot \mathrm{e}^{\alpha 1}$ 


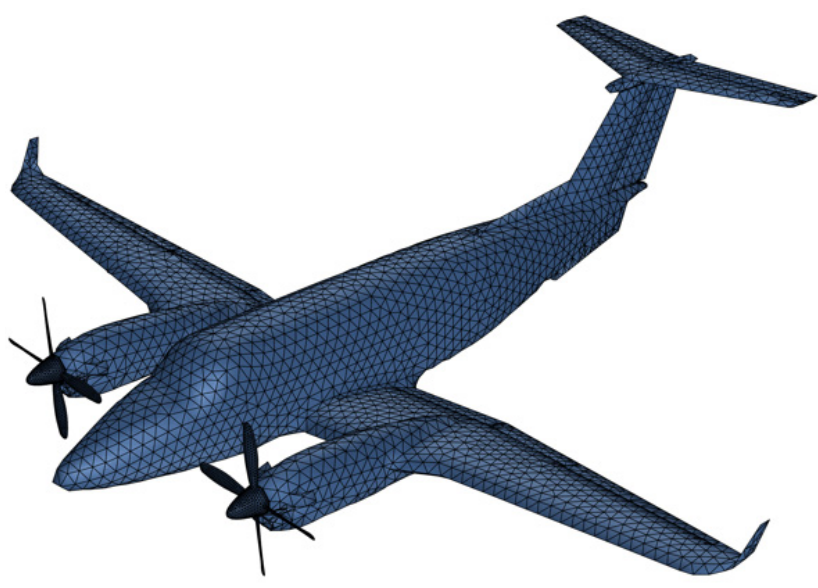

Fig. 1. Discretisation of the platform model with 15680 triangles for analysis up to $118 \mathrm{MHz}$.

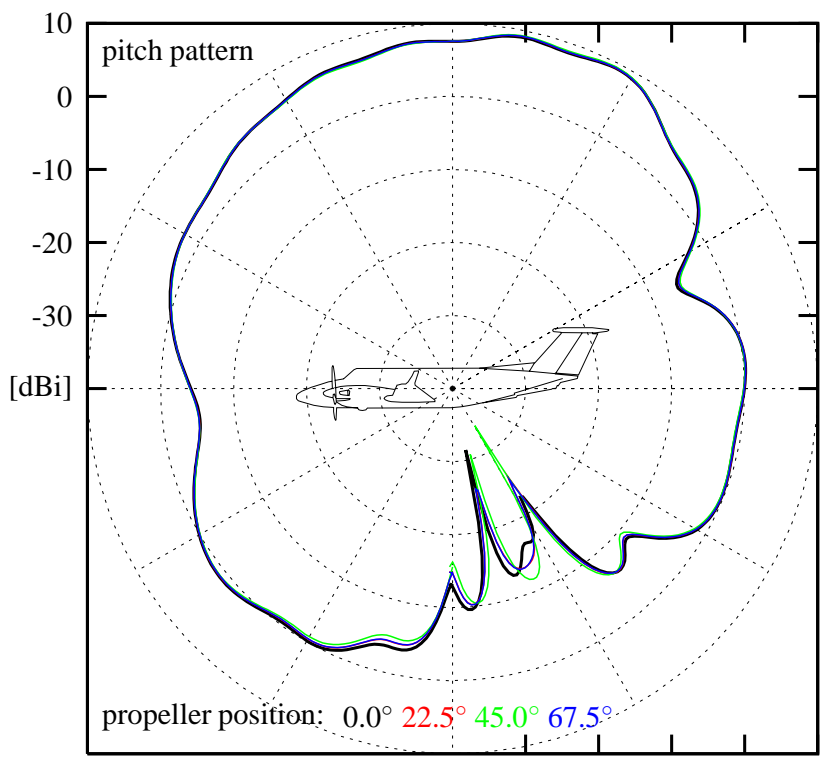

Fig. 2. Simulated pitch pattern of installed VHF top antenna at $f=113 \mathrm{MHz}$.

\section{Aircraft model and simulation}

As stated above, a numerical simulation of the installed antenna performance using the method of moments was applied. In order to obtain the antenna factor, first the gain of the antenna is computed from the radiation pattern. For most of the relevant antennas, equivalent models can be created using analog radiation characteristics, i.e. gain $G_{0}$ of the original antenna. For complex antennas with unknown impedance matching networks, the reflection coefficient $r$ can be determined from measurements.

Effectively, some relevant areas of the platform have geometrical variations within the order of the wavelength or

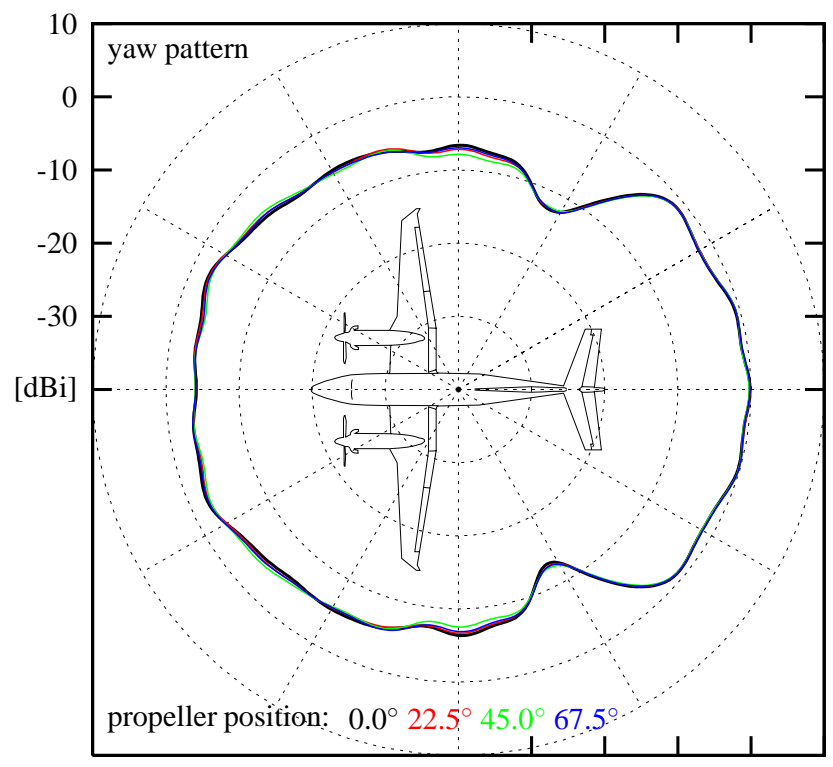

Fig. 3. Simulated yaw pattern of installed VHF top antenna at $f=113 \mathrm{MHz}$.

less (propeller blades, leading edge curvatures, surface curvatures etc.), so a full-wave solution of Maxwell's equations is required as opposed to a (less computational intensive) asymptotic solution, assuming pure ray-optical behavior of the electromagnetic field. For this work a multilevelfast-multipole accelerated method of moments (MLFMA) is used. The method of moments is a well known and established technique for antenna analysis (Harrington, 1968), while the MLFMA acceleration is a relative new addition allowing the analysis of much larger structures (Chew et al., 2000).

The method of moments requires the geometry to be meshed by elements of about a tenth of the wavelength in size. Here, triangular shaped elements are used. For the aircraft considered here, a Raytheon Beech Super King Air 350 , a total of about 16000 triangles is required to model the geometry at VHF NAV frequencies $(108-118 \mathrm{MHz})$ and of about 600000 at L-band frequencies around $1 \mathrm{GHz}$, respectively. An image of the VHF mesh is shown in Fig. 1.

Some 3-D-gain antenna patterns referencing an isotropic radiator $\left(G_{0}=1\right)$ are exemplified in the following diagrams. The VHF antenna which is mounted on top of the Beech King Air gives a pitch pattern at $113 \mathrm{MHz}$ shown in Fig. 2 and a corresponding yaw pattern in Fig. 3. In contrast, the concerned L-Band frequencies, e.g. for DME (Distance Measuring Equipment), are roughly 10 times higher than for VHF NAV so the resulting pattern has finer structures which can be seen in Figs. 4 and 5 at $1068 \mathrm{MHz}$. Since the L-band antenna is mounted underneath, the aircraft itself shields radiation in the upper direction which can be observed in the pitch pattern (Fig. 4). Relevant gain changes due to the 


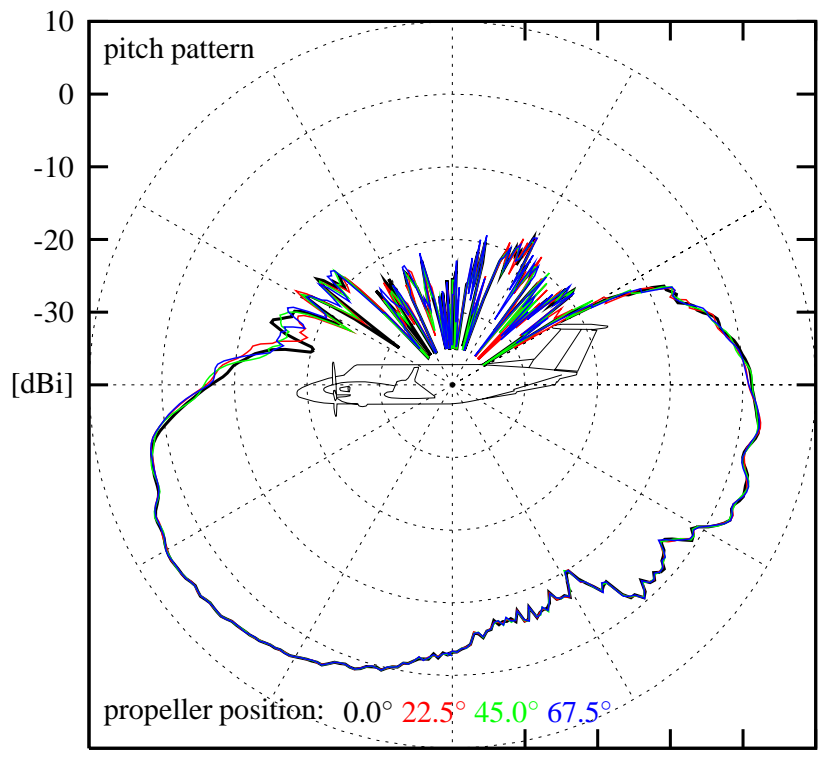

Fig. 4. Simulated pitch pattern of installed L-Band (DME) antenna at $f=1068 \mathrm{MHz}$.

4-blade-propeller position can only be observed at directions which are not relevant for the purpose of the antenna, e.g. towards ground as shown in the VHF pitch pattern shown in Fig. 2.

\section{Comparison to measurements}

In order to verify the simulation results, near field measurements of three different antennas were performed by the Physikalisch-Technische Bundesanstalt (PTB) at the airport in Braunschweig. Two of the antennas were dipole antennas for the VHF (108-118 MHz) and UHF (329-335 MHz) band installed above the cockpit whereas the third was a L-band antenna installed underneath the hull of the aircraft.

For the measurements the aircraft was positioned on a $20 \mathrm{~m} \cdot 20 \mathrm{~m}$ ground plane made up of a conductive textile. The reference antenna was positioned on a circular arc with a radius of $R=7.75 \mathrm{~m}$ facing the aircraft under an aspect angle $\alpha$, as indicated in Fig. 6, between -60 and +60 degrees. The measurements for the L-band antenna were performed in a plane $90 \mathrm{~cm}$ above ground, whereas the measurements for the UHF and VHF antennas were performed in planes at three different heights $1.5,2.5$, and $3.5 \mathrm{~m}$ above ground. A photograph of the aircraft mounted on pillars above the ground plane and of the reference antenna mast is shown in Fig. 7. The ground plane acts as a co-radiator and therefore has surface currents which are visualized exemplarily at $118 \mathrm{MHz}$ in Fig. 8.

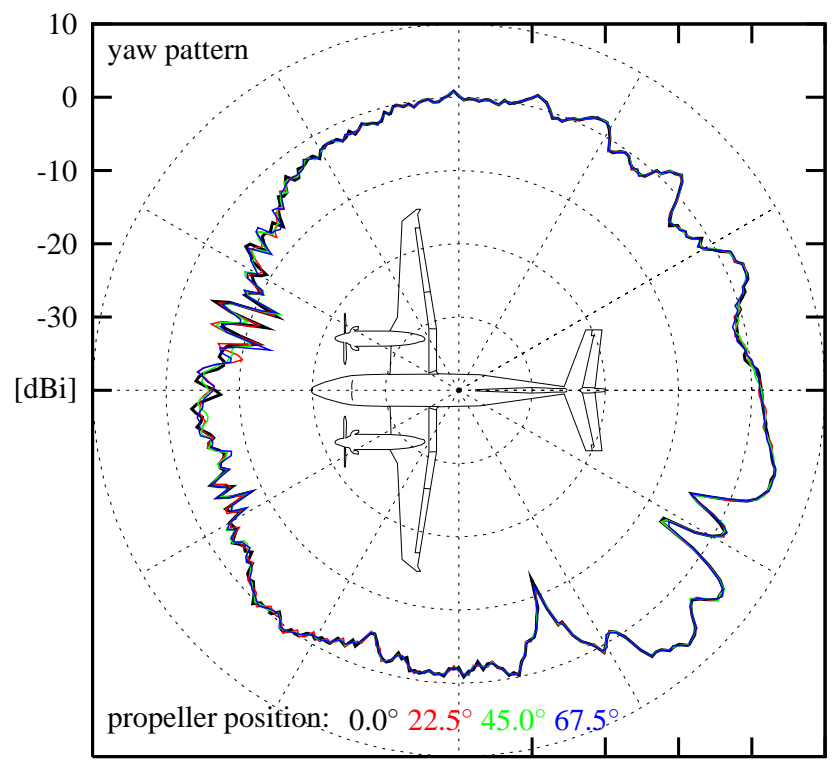

Fig. 5. Simulated yaw pattern of installed L-Band (DME) antenna at $f=1068 \mathrm{MHz}$.

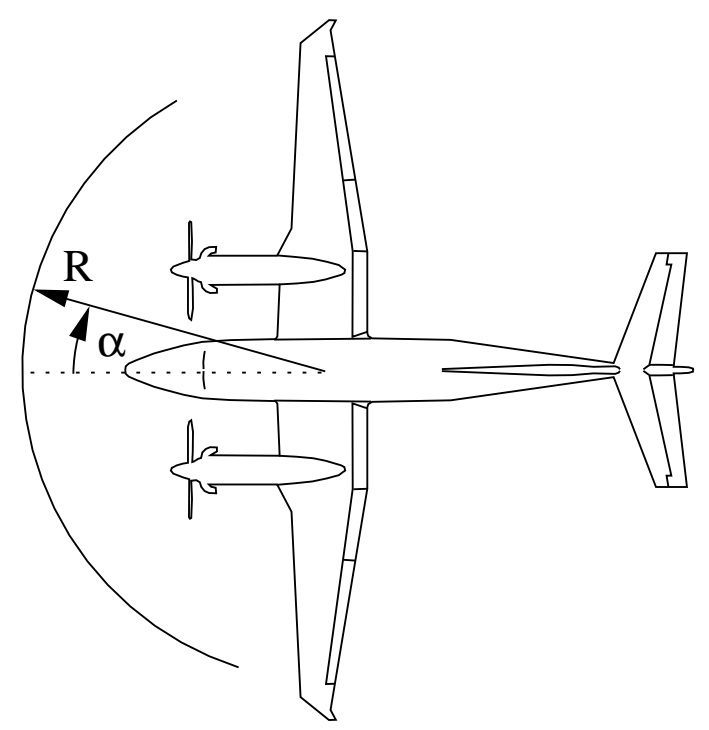

Fig. 6. Aspect angle.

\subsection{Quantity for comparison}

As quantity for the comparison we use the electrical field strength at the position of the reference antenna which has to be derived from the detected power $P_{d}$. This power is a quantity integrated over the effective aperture of the reference antenna. In order to be able to assign a detector field strength to a discrete point in space its value has to be sufficiently constant in an area comparable to the effective aperture.

If the range between transmission (aircraft) antenna and receiver (reference) antenna is considered as 2-port where 


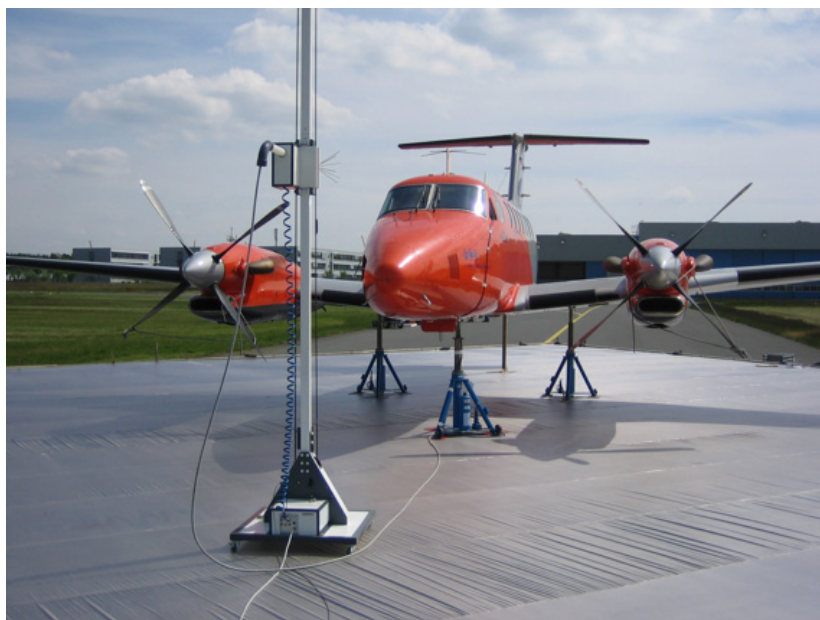

Fig. 7. Flight inspection aircraft and reference antenna mast positioned on a conductive textile ground plane.

port 1 is the transmitting antenna and port 2 is the receiving antenna, the scattering parameter $S_{21}$ describes the relation between detected power and feed power of the transmitting antenna $P_{f}$ as

$P_{d}=\left|S_{21}\right|^{2} P_{f}$.

Substituting the feed power $P_{f}$ with the expression for the radiated power

$P_{t}=\left(1-\left|S_{11}\right|^{2}\right) \mathrm{e}^{-2 \alpha l} P_{f}$,

where the first factor on the right side expresses the mismatch losses and the second factor the losses of the antenna cable with length $l$ and attenuation coefficient $\alpha$ yields

$P_{d}=\frac{\left|S_{21}\right|^{2}}{\left(1-\left|S_{11}\right|^{2}\right) \mathrm{e}^{-2 \alpha l}} P_{t}$.

The received power can be expressed as the product of radiation density in far field approximation $S=|E|^{2} / \eta_{0}$ (freespace impedance $\eta_{0}$ ) and the effective aperture of the receiver antenna $A_{r}$ by

$P_{d}=S A_{r}=\frac{|E|^{2}}{\eta_{0}} g_{r} \frac{\lambda^{2}}{4 \pi}$

where $g_{r}$ is the known reference antenna gain.

Using Eq. (8) the magnitude of the electric field strength $|E|$ at the location of the detector antenna can be calculated as

$$
|E|^{2}=\frac{\left|S_{21}\right|^{2}}{\left(1-\left|S_{11}\right|^{2}\right) \mathrm{e}^{-2 \gamma 1}} \frac{4 \pi \cdot \eta_{0}}{\lambda^{2} g_{r}} P_{t}
$$

with known radiated power $P_{t}$ and gain $g_{r}$ of the detector antenna. The feed power is set to 1 Watt to be able to compare the measurements to the simulations which have been performed for this power level.

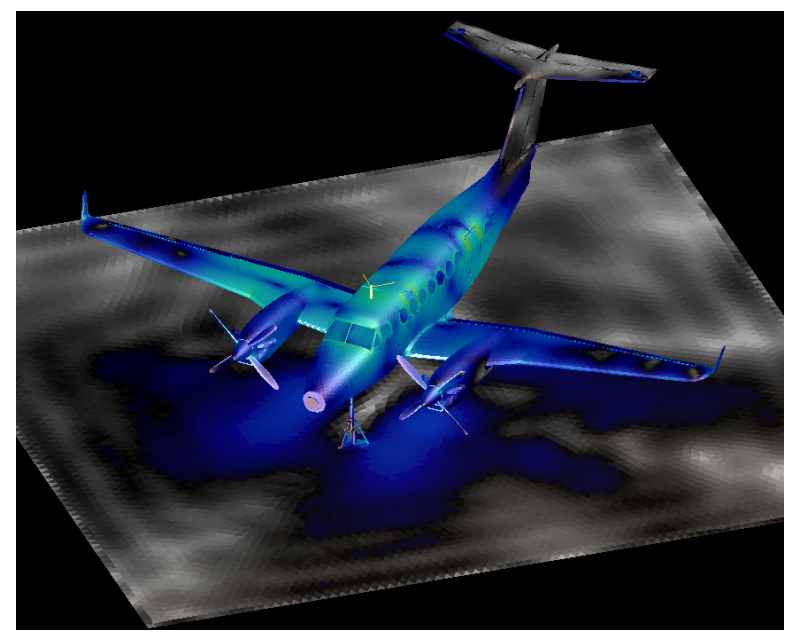

Fig. 8. Surface currents caused by the VHF NAV top antenna above a ground plane at $f=118 \mathrm{MHz}$. The weakest currents are colored white.

\subsection{VHF antenna}

The horizontally polarized V-shaped VHF dipole is located above the cockpit. According to its specifications it has a VSWR of 3:1 which corresponds to a reflection factor of $\left|S_{11}\right|=0.5$ (so mismatch loss is $1.25 \mathrm{~dB}$ ). The RG-400 antenna cable has a length of $4.68 \mathrm{~m}$ and an attenuation of $0.73 \mathrm{~dB}$ in the frequency range from 108 to $118 \mathrm{MHz}$ according to its specifications.

A fundamental reason for the discrepancy between simulation and measurement can be found in the inhomogeneity of the electromagnetic field at the location of the detector antenna. As already discussed in Sect. 3.1, a homogeneous field is the prerequisite for calculating the field strength level from the measured receiver power. Figure 9 shows the real part of the normal component of the radiation power density in comparison to the radiation power density obtained from the copolar electric field strength over the aspect angle at a height of $3.5 \mathrm{~m}$ and $f=108 \mathrm{MHz}$. Obviously the assumption of a plane wave with $=|E|^{2} / \eta_{0}$ is no longer valid which means that a precise calculation of the field strength from the received power is no longer possible.

For an estimate of the resulting error we simulate the measurement situation with a $60 \mathrm{~cm}$ dipole at $108 \mathrm{MHz}$ at a height of $3.5 \mathrm{~m}$. The dipole length is comparable to the dimensions of the actual measurement antenna. From the calculated receiver power the field strength is then determined: The dipole has an input impedance of $Z_{D}=(9.4-J 649.6) \Omega$ and a gain of $1.84 \mathrm{dBi}$ in free space. In the case of a matched load resistance of $Z_{L}=Z_{D}^{*}=(9.4+\mathrm{J}$ 649.6) $\Omega$ the field strength level determined in this way is only $0.13 \mathrm{~dB}$ below the directly simulated value without antenna. A mismatch in this impedance range has significant impact on the received power and hence on the field strength level. A load resistance 


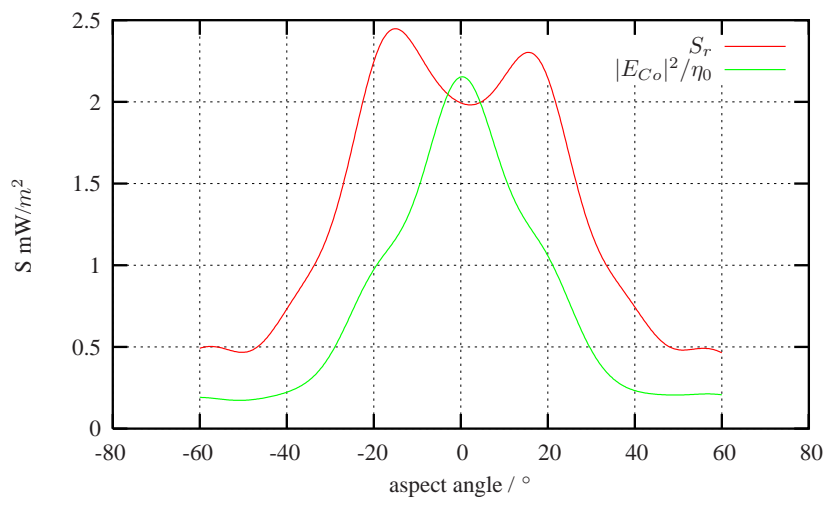

Fig. 9. Real part of the Poynting vector compared to the corresponding value derived from the copolar electric field strength.

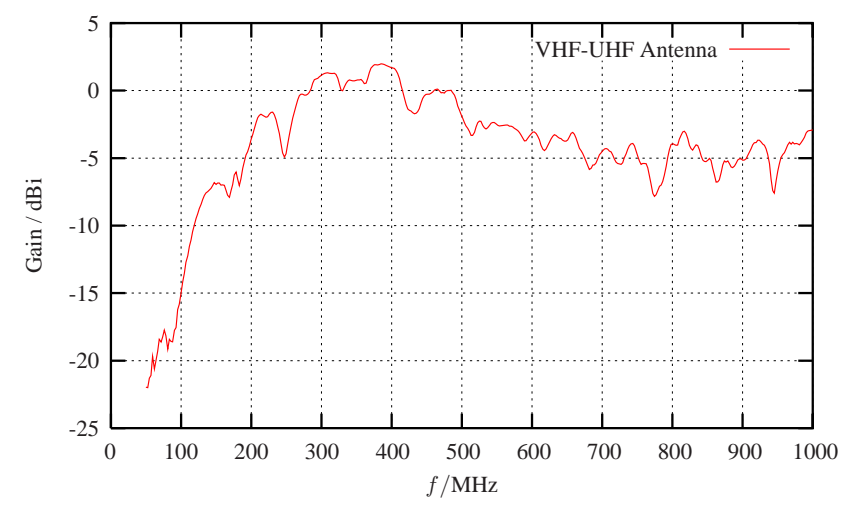

Fig. 10. Gain of the PTB reference antenna.

of $Z_{L}^{\prime}=(9.82+J 682) \Omega$ with both real and imaginary values only $5 \%$ above the matching value causes the field strength level to decrease by $6 \mathrm{~dB}$.

The reason for this sensitivity can be found in the unbalanced magnitude of real and imaginary part of the input impedance of the dipole. We assume that discrepancies between simulations and measurements can be attributed to such a situation. Figure 10 shows the measured gain of the receiver antenna over frequency. It decreases rapidly towards lower frequencies.

In order to be able to better match simulations and measurements, the measured values have been corrected by a constant offset of $2 \mathrm{~dB}$. Figures 11 and 12 show the corrected measurements (dots) and the simulations (lines) for 113 and $118 \mathrm{MHz}$ at different receiver antenna heights, respectively. For a height of $2.5 \mathrm{~m}$ a pronounced dip for a low aspect angle can be observed for both frequencies. We attribute this to the influence of the conducting ground plane. Although both simulations and measurements show this behavior, we observe the strongest discrepancy in this case, especially at $118 \mathrm{MHz}$. However, with respect to the deviations from the homogeneity of the field and the ideal conduction of the ground used in the calculations, the match between simulations and measurements is satisfactory.

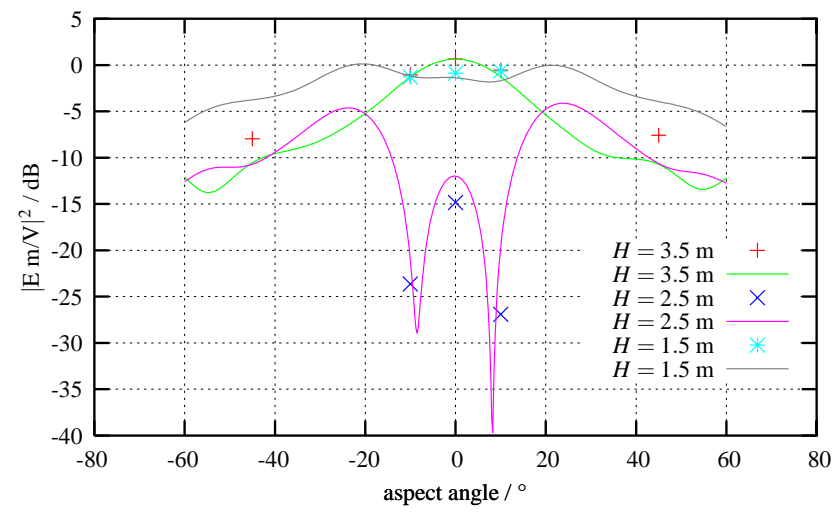

Fig. 11. Field strength level over aspect angle for different reference antenna heights at $f=113 \mathrm{MHz}$ (VHF NAV top antenna, lines: simulation).

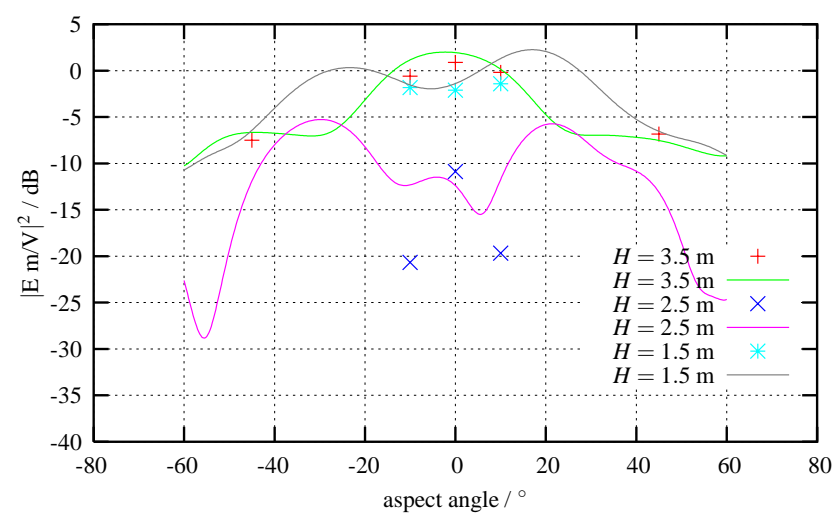

Fig. 12. Field strength level over aspect angle for different reference antenna heights at $f=118 \mathrm{MHz}$ (VHF NAV top antenna).

\subsection{UHF glideslope antenna}

The horizontally polarized UHF dipole is located in front of the V-shaped VHF dipole. According to specifications it has a VSWR of 2:1, corresponding to a reflection factor of $\left|S_{11}\right|=1 / 3$ ( $=-9.5 \mathrm{~dB}$, mismatch loss $\left.0.5 \mathrm{~dB}\right)$. The antenna is also fed via a RG-400 cable with a length of $5.12 \mathrm{~m}$ and a resulting attenuation of $1.5 \mathrm{~dB}$ at a frequency of $332 \mathrm{MHz}$ (according to data sheet).

Figure 13 shows the comparison between measurements (dots) and simulations (lines) for the transmitting UHF antenna. The agreement is much better in this case because the receiver antenna operates under much better far field conditions. Expressed in wavelengths, it is located at approximately three times the distance from the transmitting antenna compared to the previous VHF measurement.

\subsection{L-band antenna}

The L-band antenna is a monopole. It is fixed underneath the hull of the aircraft between the wings and is vertically 


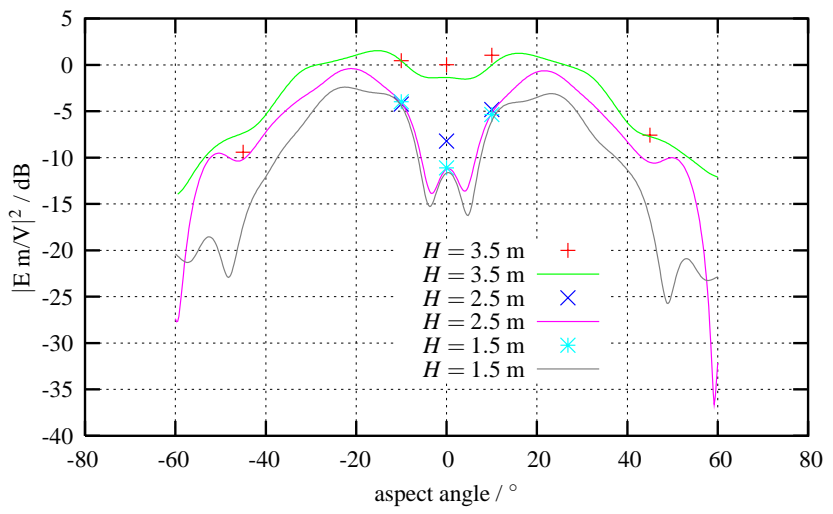

Fig. 13. Field strength level over aspect angle at different reference antenna heights at $f=332 \mathrm{MHz}$ (UHF Glideslope top antenna).

polarized. According to specifications it has a VSWR of approximately 1.5:1 which corresponds to a reflection factor of $\left|S_{11}\right|=0.2$ ( $\hat{=}-14 \mathrm{~dB}$, mismatch loss $\left.0.18 \mathrm{~dB}\right)$. The attenuation of the feeding cable is yet unknown.

Figure 14 shows the calculated and measured field strength levels plotted over aspect angle at a frequency of $1075 \mathrm{MHz}$ and at a height of $H=90 \mathrm{~cm}$ over ground.

Over a vast range of aspect angles the field strength level difference between measurements and simulations reaches up to $10 \mathrm{~dB}$. Due to the restricted number of measurement points the measurement characteristics lack from undersampling which makes it more difficult to interpret the curve characteristics. However, over small aspect angle ranges the measurements show a different trend than the simulations. A constant field strength level offset as expected from the contribution of the antenna cable attenuation which has not been considered here, would not give a satisfactory explanation.

In order to be able to evaluate the stability of the simulated model also in this case the modelling of the transition between wings and hull has been examined further. This area is close to the radiating aircraft antenna and partially non-conducting. Also the influence of the field inhomogeneity at the location of the reference antenna has been examined. This involved modelling the measurement situation where the receiver dipole, which has a size comparable to the aircraft antenna, was placed at the front aspect. The field strength level has then been calculated from the received power at the receiver antenna according to Eq. (10) and was compared to the directly determined field strength level, afterwards.

Although the field strength level calculated in this way varies with detector antenna height by $2.5 \mathrm{~dB}$ in this case, the field strength level calculated from the receiver power $P_{r}$ and the directly determined value differ by less than $0.1 \mathrm{~dB}$ at $H=90 \mathrm{~cm}$ over ground. Therefore the field inhomogeneity can be excluded as a possible reason for the difference between measurement and simulations.

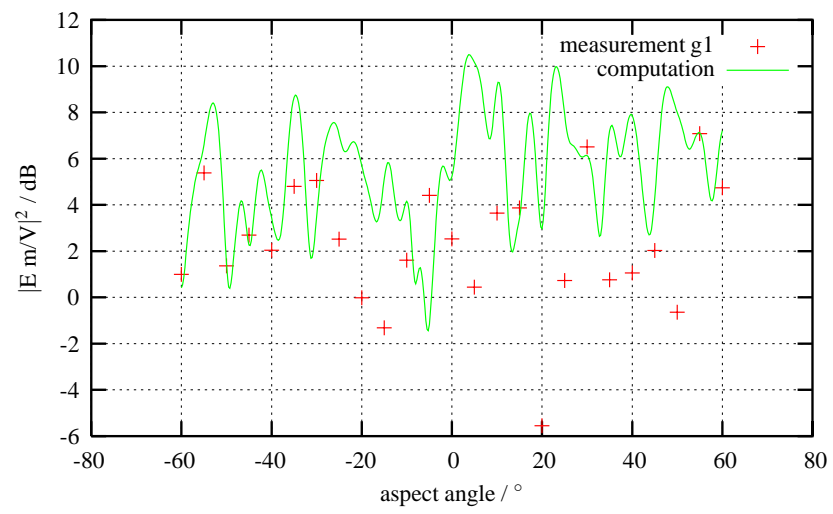

Fig. 14. Field strength level at $90 \mathrm{~cm}$ reference antenna height over aspect angle at $f=1075 \mathrm{MHz}$.

A possible explanation for the difference between measurements and simulations could be found in the realization of the conducting ground plane. The reflection characteristics of the conducting textile remains to be determined in an adequate measurement setup for both polarization types. Also the local variation in flatness of the ground plane (as can be seen in Fig. 7) is not negligible.

\subsection{Mutual L-band S-parameters}

In addition to the ground plane measurements between an aircraft antenna and a remote detector antenna in-flight measurements between mutual aircraft L-band antennas underneath the hull were performed. In order to further verify the accuracy of the computer model, three pairs of antennas were measured. The amplitude of transmission factors $\left|S_{21}\right|$ were compared to simulated data obtained from the MLFMA model of the aircraft for the respective antennas.

The measurements have been performed during an $1 \mathrm{~h}$ test flight over Northern Germany using a HP8753D vector network analyzer. The measurements were performed at an altitude of $5000 \mathrm{ft}$. with a cloud ceiling far underneath and above the aircraft. The transponder was switched off to prevent unwanted interference with the measurements. After TOSM calibration of the vector network analyzer at the end of the two $2.5 \mathrm{~m}$ long measurement cables and after the consecutive measurements of the antennas under test the performance of the network analyzer was checked based on the $E_{n}$ criterium (EAL, 1996). Measurements of calibrated PTB precision attenuators $(10 \mathrm{~dB}, 20 \mathrm{~dB}, 30 \mathrm{~dB}$ and $50 \mathrm{~dB})$ proved that the antenna measurements in between are within the expected measurement uncertainty of less than $0.25 \mathrm{~dB}$.

Figure 15 shows the result of the measurements between the previously described L-band antenna centered between the wings used as transmission antenna (no. 23 in numeration of all aircraft antennas) and three other L-band antennas used as detector antennas (no. 26 underneath the right wing, no. 25 underneath the left wing and antenna 15 underneath 


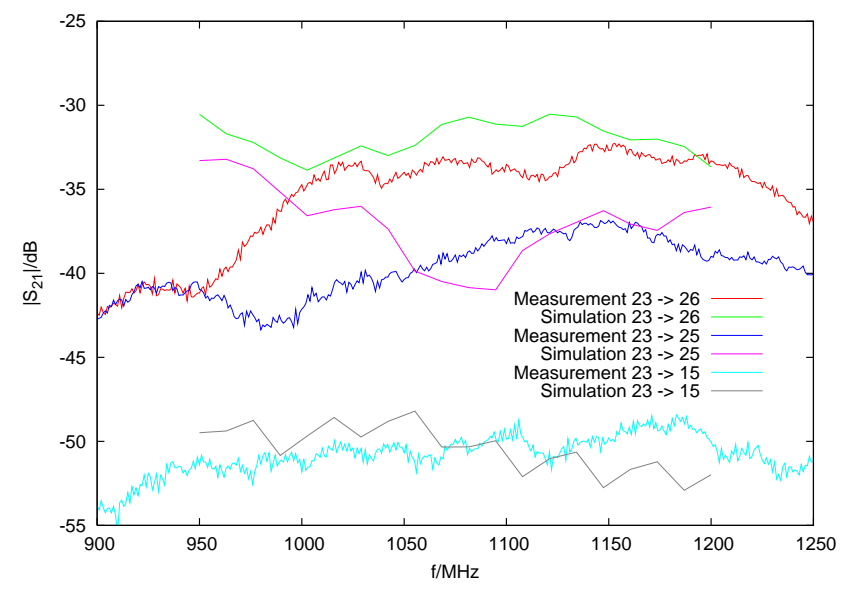

Fig. 15. Comparison of simulated and measured S-parameters of L-band antennas. The numbers 15, 23, 26 according to list of all aircraft antennas.

the rear hull). Measurements and calculations agree well in all cases but especially for the antenna pairs $23-25$ and 2315. The calculated attenuation has the tendency to be weaker than the measured values. We attribute this to the attenuation of several meters of RF cable which have been neglected so far. Further investigations to incorporate cable attenuation measurements into the calculation of effective antenna factors are underway.

\section{Conclusions}

We showed that a balanced combination of antenna measurements, platform modelling and MoM simulations is the key to obtain the effective antenna installed performance. However, some problems impede the comparison of measurement results and numerical solutions under certain circumstances: The VHF results were gained in a near field environment whereas the L-band antennas underneath the aircraft did not meet the ideal conditions above the ground plane as taken for granted in the model. In the VHF case one has to consider alternative measurement concepts. A solution could be to implement a near field scanner in context with adequate near to far field transformations. Further work is needed to implement such technologies and the authors will continue their work in upcoming projects.

Acknowledgements. The authors would like to thank M. Schwendener and J. Follop of Flight Calibration Services for their assistance during the measurement campaigns and J. Reiter of EADS for his contributions to the simulations.

\section{References}

Balanis, C. A.: Antenna Theory Analysis and Design, Wiley, New York, 1997.

Bredemeyer, J., Battermann, S., Garbe, H., and Ritter, J.: Antenna Installed Performance of a Flight Inspection Aircraft, Proceedings of International Symposium on Precision Approach and Automatic Landing (ISPA 2004), Munich, Germany, 2004.

Chew, W. C., Jin, J.-M., Michielssen, E., and Song, J. M.: Fast and efficient algorithms in computational electromagnetics, Artech House, Boston, London, 2000.

DIN EN 45003 (Akkreditierungssysteme für Kalibrier- und Prüflaboratorien; allgemeine Anforderungen für Betrieb und Anerkennung), 1995.

International Civil Aviation Organization: DOC 8071, Manual on Testing of Radio Navigation Aids, Volume I: Testing of Groundbased Radio Navigation Systems, Montreal, 2000.

European cooperation for Accreditation of Laboratories, Publication Reference EAL-P7, EAL Interlaboratory Comparisons, Edition 1, March, 1996.

Harrington, R. F.: Field Computation by Moment Methods, Cazenovia, N.Y., USA, 1968.

Kraus, J. D.: Antennas, Mc-Graw-Hill, Boston, 1988.

Unger, H.-G.: Elektromagnetische Wellen auf Leitungen, 4. Aufl., Heidelberg, 1996. 